\title{
Sedimentation speed of inertial particles in laminar and turbulent flows
}

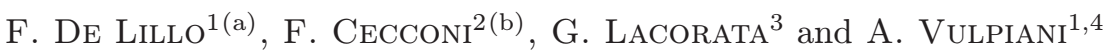 \\ ${ }^{1}$ Dipartimento di Fisica, Università di Roma "La Sapienza" - P.le Aldo Moro 5, 00185 Roma, Italy, EU \\ ${ }^{2}$ Center for Statistical Mechanics and Complexity (SMC) INFM-CNR and Istituto dei Sistemi Complessi \\ (ISC-CNR) - Via dei Taurini 1900185 Roma, Italy, EU \\ ${ }^{3}$ Istituto di Scienze dell'Atmosfera e del Clima, CNR - V. Monteroni, 73100 Lecce, Italy, EU \\ ${ }^{4}$ INFN sezione Università di Roma "La Sapienza" - Roma, Italy, EU
}

received 3 April 2008; accepted in final form 9 October 2008

published online 4 November 2008

PACS 05.45.-a - Nonlinear dynamics and chaos

PACS $47.52 .+j-$ Chaos in fluid dynamics

PACS 47.57. ef - Sedimentation and migration

\begin{abstract}
We consider passive inertial particles settling under the combined action of gravity and a Stokes drag in two-dimensional velocity fields with increasing complexity. The average settling speed is studied in simple steady and time-dependent cellular and in turbulent flows. Different regimes are identified in relation to the physical properties and complexity of the fields. While for steady and slowly varying weak cellular fields the settling velocity is a decreasing quadratic function of the Eulerian velocity, the same behaviour does not occur for actual turbulent fields. Large fluid velocities, on the other hand, generally speeds up the settling process. The relationships between settling times and the properties of particle trajectories in the different regimes are discussed.
\end{abstract}

Copyright (c) EPLA, 2008

Introduction. - Many applications of fluid mechanics involve the transport of particles of non-negligible size and mass. Typical examples are pollutants in fluids, settling of solid particles, rain formation in clouds, and fuel jets in diesel engines [1-3]. Even in the case of very dilute suspensions, where the feed-back of the solid phase on the fluid can be neglected, the behaviour of inertial particles strongly differs from that of fluid ones. A typical effect due to inertia is preferential concentration: inertial particles tend to accumulate in certain regions of the flow, even if the velocity field is divergenceless [4]. The details of particle distributions and of the related statistics are still the object of theoretical [5-7], experimental $[8,9]$ and numerical investigation $[6,10-15]$. A closely related problem is that of determining the average settling speed of inertial particles. This problem has a relevance in atmospheric and climate research (in particular, for the physics of clouds and rain formation $[2,3])$ and in marine biology (e.g., to understand the dynamics of plankton populations [16]). Since the original work by Stommel [17],

(a) Present address: Dipartimento di Fisica Generale, Università di Torino - Via Giuria 1, 10125 Torino, Italy, EU.

(b) E-mail: fabio.cecconi@roma1.infn.it several authors have addressed the problem of settling inertial particles by considering the effect of vortical structures, modelled as a periodic array of counterrotating cells. This approach was aimed at clarifying the possibility of particle entrapment by coherent flow structures, which could eventually lead to a decrease in sedimentation velocity. Maxey and Corrsin [18] studied the motion of small heavy particles settling through an infinite, periodic, cellular flow field [19]. Particle inertia was shown to have an important influence on the motion: no permanent suspension in the flow occurred, particles generally settled more rapidly than in still fluid, with a decrease in the settling velocity only observed for large inertia. The authors of that study also observed the tendency of particles with low inertia to fall along the downward sides of cells. Analogous investigations have been carried out in the case of particles lighter than the fluid (such as air bubbles in water), that tend to raise due to negative buoyancy $[20,21]$. In both cases preferential concentration has a strong effect on settling. Both numerical $[22,23]$ and experimental $[24,25]$ results indicate that the settling velocity is increased by homogeneous turbulence. However in synthetic turbulent flows, for certain values of the parameters, the sedimentation speed can vanish [26]. 
In this paper we are interested in the effect of the velocity field $\mathbf{U}=\left(U_{x}(x, y, t), U_{y}(x, y, t)\right)$ on the sedimentation process, without taking into account the interaction among the particles and the feedback on the fluid. We consider the case of very heavy particles, for which the only relevant contribution is the Stokes drag. The suspension is assumed to be diluted enough not to affect the velocity field. The resulting equations are [27]

$$
\left\{\begin{array}{l}
\dot{x}=v_{x}, \\
\dot{y}=v_{y}, \\
\dot{v}_{x}=\frac{\left[U_{x}(x, y)-v_{x}\right]}{\tau}, \\
\dot{v}_{y}=\frac{\left[U_{y}(x, y)-v_{y}\right]}{\tau}-g,
\end{array}\right.
$$

where $\tau$ is the Stokes time of the particle, and $g$ is the rescaled gravitational constant. The average settling velocity is defined as $V_{s}=\left\langle H / t_{s}\right\rangle$, where $t_{s}$ is the time it takes for a particle to cover the fixed vertical distance $H$ (depth of the fluid), and $\langle\ldots\rangle$ is the average over the initial conditions. In all considered cases, $H$ is taken large enough to make initial transients negligible. When the fluid is still, the settling velocity is trivially $V_{s}=V_{0}=g \tau$. If the typical velocity and the characteristic length of the flow are $U_{0}$ and $L$, respectively, the dynamics of the system is determined by two dimensionless parameters: the Stokes number $\mathrm{St}=\tau U_{0} / L$, measuring the ratio between inertial and advective times on scale $L$, and the Froude number $\mathrm{Fr}=U_{0} / \sqrt{g L}$, measuring the ratio between advection and gravity on the scale $L$. We consider the system of units in which $\tau=1$ and $g=1$, velocities are measured in units of $V_{0}$ and lengths in units of $g \tau^{2}$ : in such units we have $V_{0}=1, \mathrm{St}=U_{0} / L$ and $\operatorname{Fr}=U_{0} / \sqrt{L}$.

In the following we study the settling of inertial particles driven by flows of increasing disorder. We consider, first, the simplest case of a laminar steady and time-dependent cellular flows. Then, we study the settling process in cellular flows with certain elements of randomness and finally we compare the results to the turbulent case.

Steady cellular flow. - The first case we consider is that of particles moving in a two-dimensional, divergenceless cellular flow of period $L$, defined by the stream function $\Psi(x, y)$

$$
\Psi(x, y)=\frac{U_{0}}{k} \sin \left(k x-\phi_{x}\right) \sin \left(k y-\phi_{y}\right)
$$

through the relations $U_{x}=\partial_{y} \Psi$ and $U_{y}=-\partial_{x} \Psi$, where $U_{0}$, $k=2 \pi / L, \phi_{x, y}$ are the amplitude, wave vector and phases characterizing the velocity field, respectively. Although apparently rather simple, the cellular model (2) (and its time-dependent generalizations, see below) is able to produce a non-trivial behaviour when advection-diffusion and Lagrangian dynamics (for fluid or inertial particles) are considered: transport features in such a field have been intensively investigated $[19,21,28]$.
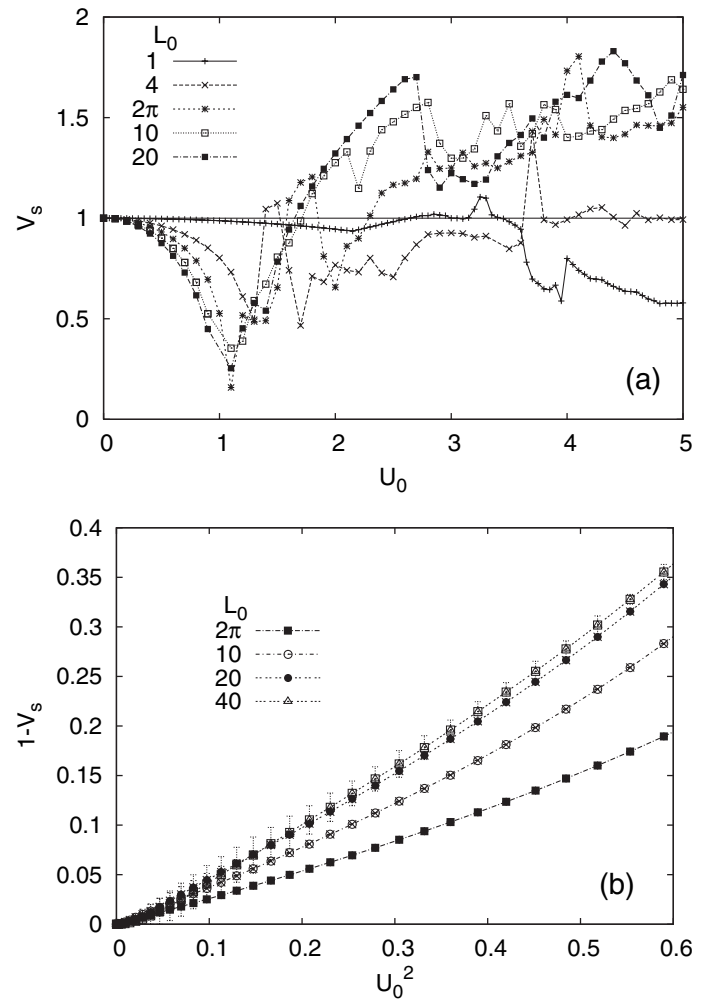

Fig. 1: In (a) behaviour of the settling velocity as a function of the amplitude of the steady cellular velocity field. The curves refer to different cell sizes. In (b) the function $1-V_{s}$ is plotted against $U_{0}^{2}$, to emphasize the predicted quadratic law (3) (only the small $U_{0}$ regime is shown). It is worth noting the collapse of the curves at large cell sizes.

Let us first present a simple qualitative argument on the effect of the velocity field on $V_{s}$. If the driving field is not too intense, i.e. $U_{0} \ll g \tau$, a falling particle is expected to be only slightly deviated from a vertical trajectory, along which it alternately encounters regions, of the order of the advective spatial scale, with upward (unfavourable) and downward (favourable) velocities, of typical magnitudes $+U_{0}$ and $-U_{0}$, respectively. Each time the particle crosses one of these regions, it attains a velocity $v_{ \pm}=g \tau \pm \alpha U_{0}$, where $\alpha<1$ is some factor resulting from the details of the flow field. The crossing time of an up-flow or down-flow region, each of length $L / 2$, will therefore be $t_{ \pm}=L /\left[2\left(g \tau \pm \alpha U_{0}\right)\right]$, thus yielding

$$
\frac{V_{s}}{V_{0}}=\frac{L}{V_{0}\left(t_{+}+t_{-}\right)} \sim 1-\alpha^{2}\left(\frac{U_{0}}{V_{0}}\right)^{2} .
$$

This simple argument predicts a parabolic decrease of the settling velocity $V_{s}$ as a function of $U_{0}$.

We tested this argument by numerical integration of eq. (1) via a 4-th order Runge-Kutta algorithm with time step $h=10^{-3} L / U_{0}$. All particles, at $t=0$, have zero velocity and are randomly distributed in one of the cells at the top of the fluid. The particle trajectories are followed until they reach a depth $H$ much larger than a cell size $L$. 

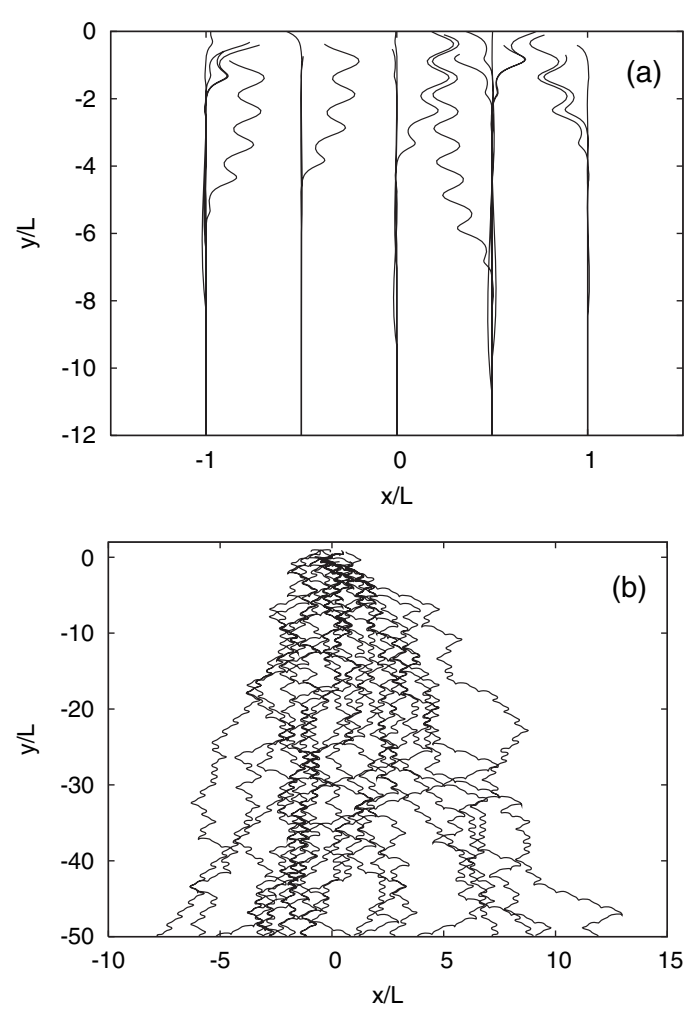

Fig. 2: Typical trajectories in the different regimes of $V_{s}$, for $L=2 \pi$. At small values of $U_{0}$ (a) trajectories collapse on the separatrices between vortices and fall vertically. This produces the initial parabolic decrease in $V_{s}\left(U_{0}\right)$. For $U_{0}>1$ (b) the trajectories become chaotic, and eventually diffuse between the cells. The plots refer to $U_{0}=0.7$ (a) and $U_{0}=2.9$ (b), respectively. Lengths are in units of the cell size $L$.

Figure 1 shows that the behaviour described by eq. (3) is actually observed, for small values of $U_{0}$. We emphasize that, based on the previous definitions, $U_{0} / V_{0}=\mathrm{Fr}^{2} / \mathrm{St}$, therefore eq. (3) is valid for relatively large inertia, in agreement with the results in [18].

When $L$ is varied, the scenario remains qualitatively unchanged. If $L$ is much larger than $g \tau^{2}=1$, the decreasing behaviour of $V_{s}$ does not depend on $L$. A large value of $L$, indeed, increases the crossing times $t_{ \pm}$, allowing the particle to closely attain the asymptotic velocity $g \tau \pm U_{0}$, and therefore producing the maximum slowing effect. Under this condition, for $U_{0} \simeq g \tau$ a particle can virtually remain suspended in the flow. When this occurs, the vertical trajectories are no longer stable. For higher values of $U_{0}, V_{s}$ increases, eventually becoming greater than 1 . The minimum is located at $U_{0} \simeq 1$ for large cells while, for smaller cells, it tends to shift towards larger $U_{0}$. The reason of this shift can be explained by noting that, at decreasing crossing times, a more intense stirring is needed to efficiently balance the particle's fall. When $L \sim g \tau^{2}$, or smaller, the shorter crossing times limit the fluctuations in particle velocity and the decrease of $V_{s}$ with $U_{0}$ becomes less and less sharp.
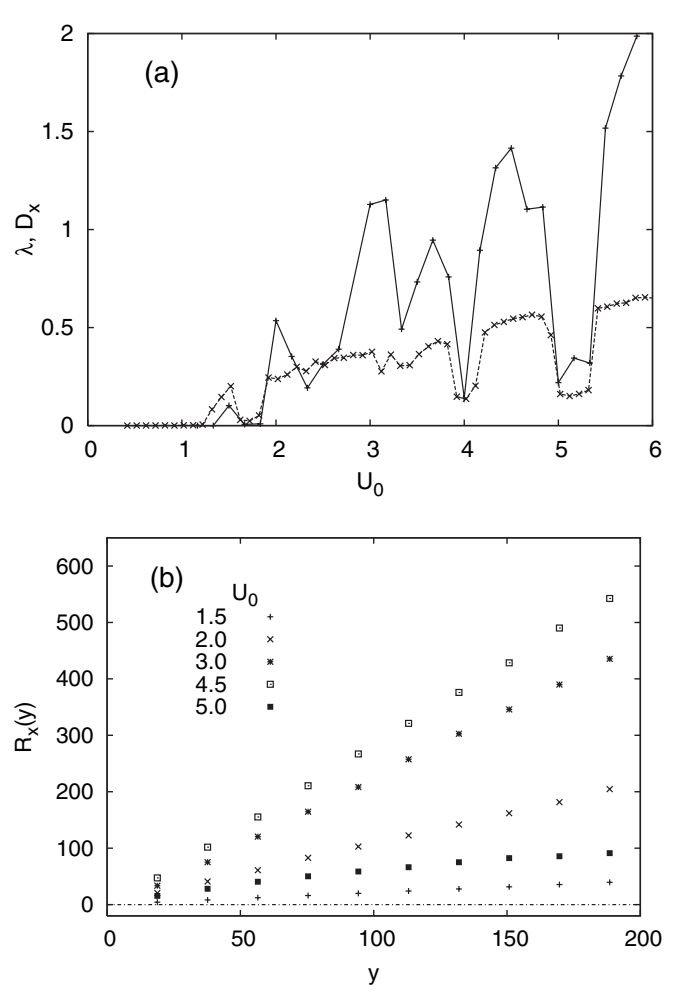

Fig. 3: Horizontal diffusion and Lyapunov exponent of particle trajectories at $L=2 \pi$. (a) The diffusion coefficient $D_{x}$ $(+)$ is plotted as a function of $U_{0}$, and compared to the Lyapunov exponent $(\times)$. (b) Horizontal dispersion $R_{x}(y)$. Different symbols correspond to various $U_{0}$. At most values of $U_{0}>1$, a linear dependence $R_{x}=2 D_{x} y$ is observed. While diffusion is absent at $U_{0} \lesssim 1$, for stronger velocity fields, a lateral diffusion of the trajectories is observed, accompanied with a positive Lyapunov exponent. The connection between chaoticity and lateral diffusion is also evident from the comparison of the curves at larger values of $U_{0}$.

An example of the influence of $U_{0}$ on particle trajectories is given in fig. 2. While for small $U_{0}$ the trajectories falling along the separatrices of the cellular structure are stable, for intermediate values of $U_{0}$ they become unstable, thus strongly reducing the contribution of the up-flow regions. At even larger values of $U_{0}$ another transition occurs, as the particles start following pinballlike trajectories which sample the down-flow regions exclusively, thus receiving only positive contributions from the flow.

Figure 3(a) indeed shows that the Lyapunov exponent of particle trajectories becomes positive in coincidence with the minimum of $V_{s}$. For $U_{0}$ large enough, trajectories can basically follow the lattice of cells in such a way to select the downward channels, reaching velocities larger than $g \tau$ (still fluid).

Lateral diffusion. - The transition between the different regimes in $V_{s}\left(U_{0}\right)$ is connected with a qualitative change in the motion of particles. It is worth noting that, beyond the "parabolic regime", one observes a lateral 
displacement of trajectories. Such a behaviour can be quantified by introducing a suitable "spatial" diffusion coefficient $D_{x}=R_{x}(y) /(2 y)$, where

$$
R_{x}(y)=\frac{1}{N} \sum_{i=1}^{N}\left[\tilde{x}_{i}(y)-\tilde{x}_{i}(0)\right]^{2}
$$

is the mean square displacement from the initial position, as a function of the vertical position (instead of being a function of time), where $\tilde{x}_{i}(y)$ indicates the position of the $i$-th particle when $y_{i}(t)$ crosses $y$ for the first time. In practice, the spreading of trajectories is measured at fixed vertical thresholds. In fig. 3 , the results are given for $L=2 \pi$. While for $U_{0} \lesssim 1$ there is no lateral diffusion, for larger velocities a non-zero $D_{x}$ is observed, as the signature of a wide displacement of particles between the cells. We note that the diffusion coefficient is non-monotonic with $U_{0}$, and presents a very irregular behaviour. Analysis of the Lyapunov exponents shows a clear connection between $\lambda$ and $D_{x}$ (see fig. $3(\mathrm{~b})$ ), with a strong decrease in lateral diffusion corresponding to drops in the chaoticity of the trajectories. The fact that the chaotic character of the system changes in a non-trivial way as a function of a parameter (in this case, $U_{0}$ ) is not surprising, and is a common feature in low-dimensional chaotic systems. For example, one can expect the Lyapunov exponent to vanish for some $U_{0}$ for which the motion of the particles is in resonance with the cellular flow resulting in regular trajectories. In the following sections we will show how adding a time-dependent component to the flow changes this behaviour.

The effects of non-stationarity. - Since the steady cellular flow is too crude a model to describe most natural flows, we considered some progressive modifications thereof, to test the robustness of the mechanism previously described. As a first generalization we study a field where the centres of the cells wander as a random walk with diffusion coefficient $D_{0}$, i.e. in eq. (2) $\left\langle\phi_{x, y}^{2}\right\rangle=2 D_{0} t$. The characteristic time of such a diffusive process is $t_{c}=4 \pi / D_{0}$ that must be compared to the typical crossing time of a cell $L / g \tau$ in order to identify different regimes. If $t_{c} \gg L / g \tau$ the particles should easily follow the slow motion of the cells and the scenario with respect to the steady field should remain practically unchanged. Simulations were carried out for different values of the diffusion constant. As fig. 4 shows, when the cells move with a very long typical time $t_{c}=10^{3}$, settling displays little difference with respect to the steady case, while for a larger diffusion coefficient $\left(t_{c} \sim 10\right)$ particle trajectories are too stiff to follow the structures of the flow and the effects of upward regions are strongly suppressed, as well as the preferential concentration on downward channels: as a consequence the settling velocity does not vary strongly from the still-fluid value. As a side remark, it is apparent that the application to the system of some sort of disturbance suppresses the irregular behaviour which
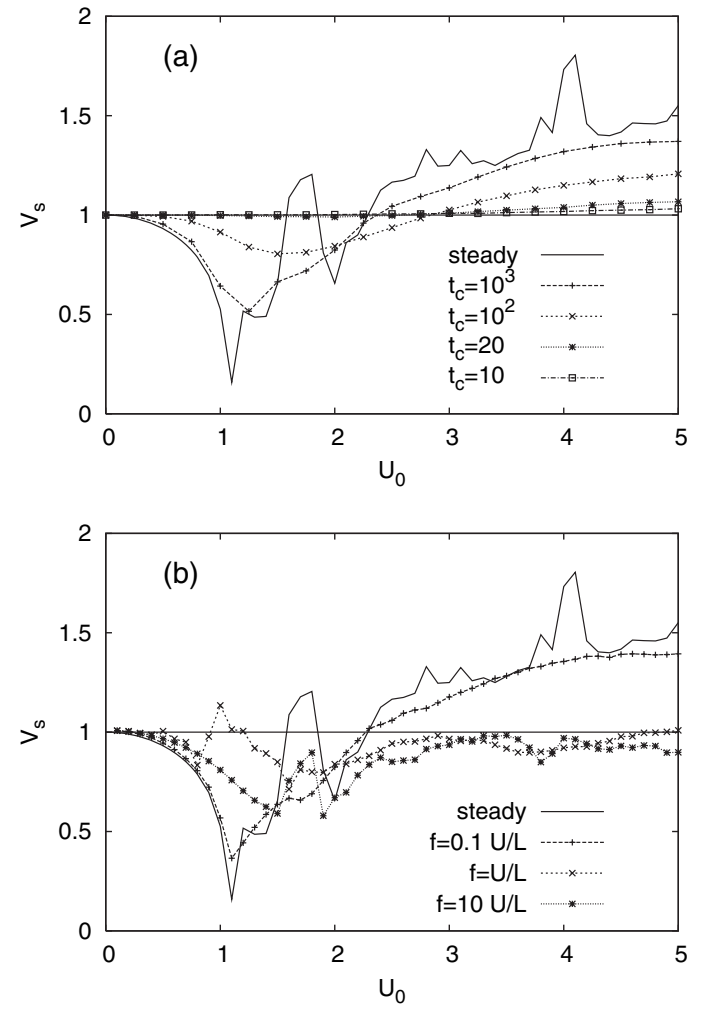

Fig. 4: (a) Behaviour of the settling velocity as a function of the amplitude of the cellular velocity field where the center of the cells undergoes diffusive random walk. $t_{c}$ indicates the typical correlation time of the flow. For large values of $t_{c}$ the main properties of the steady case are preserved, while the variation of $V_{s}$ with $U_{0}$ is strongly reduced for shorter correlation times. (b) Settling velocity of particles in a cellular velocity field oscillating with frequency $f$. The cell size is $L=2 \pi$ and the amplitude of the oscillations is $\epsilon=0.1 \mathrm{~L}$.

is evident in the curves of fig. 1 and very likely due to the extreme regularity of the steady cellular pattern.

As a further test we let the centres of the cells oscillate periodically with a frequency $f$ around the positions of the static field, $\phi_{x}=k B \cos (2 \pi f t)$ in eq. (2). As long as $f^{-1} \gg L / U_{0}$ (i.e., if the period of the oscillations is much larger than the period of the harmonic oscillation of a test particle around the stable fixed point of the steady flow) the particles can adjust their motion to that of the fluid, while in the opposite limit the flow has on the particles the effect of a random noise. In panel (b) of fig. 4 we plot the settling velocity in an oscillating cellular fluid at different frequencies. The results confirm the expected behaviour, since for small frequencies the $U_{0}<1$ regime is unchanged, while for larger $f$ the curve tends to become flat. An analysis of the lateral diffusion of trajectories was carried out also for the oscillating cellular flow, giving results qualitatively similar to the ones discussed for the stationary case.

Turbulent flow. - We repeated the computations by replacing the cellular flow with a time-dependent solution 


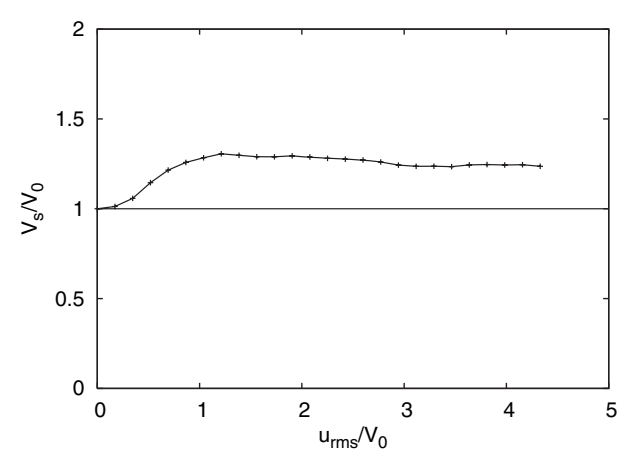

Fig. 5: Settling velocity of particles falling through a turbulent field, as a function of $u_{r m s} / V_{0}$. The increase in the settling velocities for weak velocity fields (compared to $V_{0}$ ) shows that most particles "select" paths in the flow field that are favourable for the settling process.

of the two-dimensional Navier-Stokes equations, resulting from a direct numerical simulation in the regime of direct enstrophy cascade [29]. This regime is characterized by spatially smooth velocities, in which the dynamics is strongly dominated by the large scales, and is therefore a rather natural generalization of the cellular fields considered above. The fields were obtained through a standard pseudo-spectral code [30] on a doubly periodic domain of side $L=2 \pi$, by forcing the velocity at large scale with a Gaussian random forcing, $\delta$-correlated in time, on wave numbers of modulus $|\mathbf{k}| \leqslant 2$.

We characterize the velocity field by its root mean square value $u_{r m s}$, and consequently define the dimensionless parameters $\mathrm{Fr}=u_{r m s} / \sqrt{g L}$ and $\mathrm{St}=u_{r m s} \tau / L$. The latter definition amounts to considering the large scale time as the relevant one for the advection of particles. This assumption is not valid in general and is justified here because of the properties of the smooth fields considered. However, in fully developed turbulence the Kolmogorov time (i.e. the typical time of dissipative scales) is a more appropriate choice (e.g., in [13]). In the turbulent case we rescaled the particle parameters instead of the fluid velocity. By the rescaling $\tau^{\prime}=\alpha \tau, g^{\prime}=\rightarrow g / \alpha^{2}$, one obtains $u_{r m s} / V_{0}^{\prime}=\alpha u_{r m s} / V_{0}$, which is equivalent to a rescaling of the velocity by $\alpha$. The Stokes and Froude numbers rescale accordingly. At variance with the cellular flow no decrease in $V_{s}$ is observed at $u_{r m s} \lesssim 1$. The selection of downward channels is dominating, producing an increase of the settling speed with $u_{r m s}$ (see fig. 5). One natural simplification of the problem of settling in turbulent flows is to consider steady turbulent or turbulencelike fields. Such a field can be considered as a random superposition of modes at different scales. The hypothesis that guides the attempts in this direction is therefore that this kind of field possesses a sufficient complexity to reproduce the properties of the time-dependent field even when time dependence is suppressed. We carried out computations using fixed snapshots from our turbulent simulations. The results varied greatly depending on the
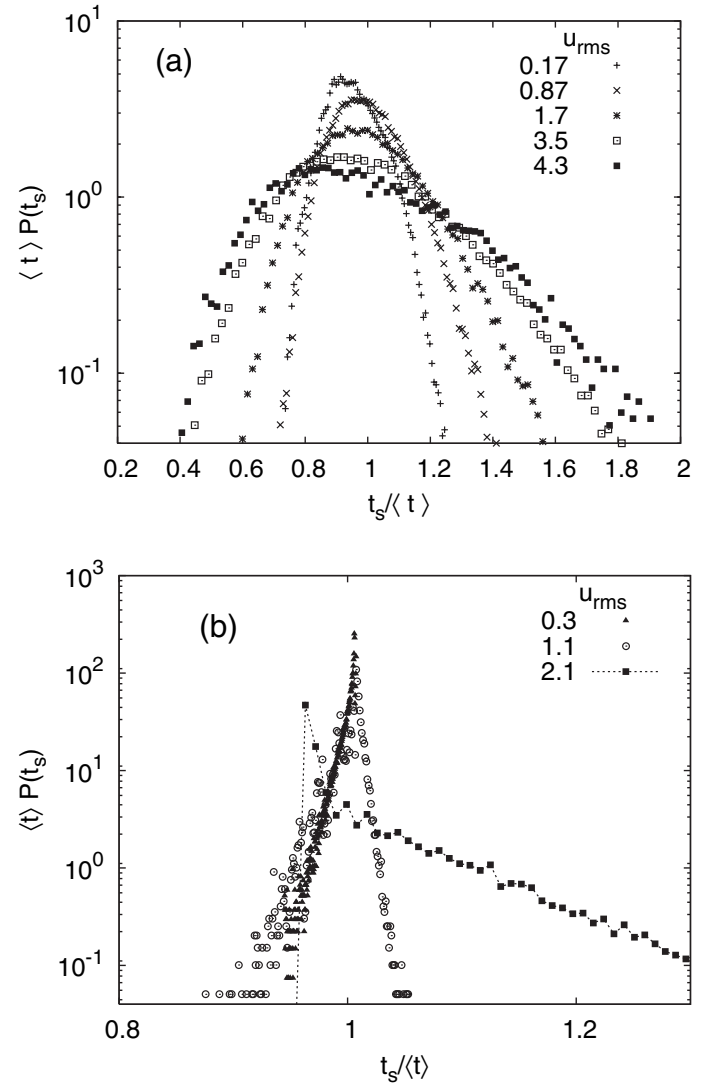

Fig. 6: Probability distribution of the settling time $t_{s}$ for various values of the velocity field amplitude. The two panels (a) and (b) refer to turbulent and cellular flow, respectively. Both figures indicates that the $t_{s}$ distributions become wider and wider as the amplitude of the flow increases. Data are rescaled with the average to allow a direct comparison on the same scale.

details of the chosen configuration. In particular, comparatively more regular fields, characterized by well-defined large-scale vortices, displayed a behaviour similar to the one observed in cellular flow, with a decrease in $V_{s}$ for $u_{r m s} \lesssim 1$. However, other snapshots produced a monotonic increase of the settling velocity with $u_{r m s}$. The phenomenology of settling on stationary fields therefore cannot be parametrized by simple quantities, like the rms velocity, and is strongly non-universal, at least when few scales are involved. The results obtained with time-dependent fields, where no slowing-down of settling is observed, show that the evolution in shape of flow structures produces a faster settling. This observation is compatible with the results in [26], where static velocity configurations were shown to produce particle trapping, which disappears with time-dependent field configurations.

In ref. [31] the authors observe a non-monotonic dependence of the settling velocity on St. However that study focuses on the limit of small St, which in a sense is complementary to the one considered here.

Sedimentation time statistics. - As further comparison between cellular and turbulent fields, we 
considered the distribution of the sedimentation time $t_{s}$. At this regard, we computed the statistics of $t_{s}$ defined as the first exit time from the bottom boundary $y=-H$, both in the cases of cellular and turbulent flows (fig. 6). The cellular flow, for small $U_{0}$, is characterized by a smooth exit time distribution. For $U_{0} \sim g \tau$, the PDF displays a peaked structure due to preferential concentration along few channels corresponding to the separatrices of the cellular flows. For larger values of $U_{0}$, the trajectories spread over more and more channels so that the distributions are characterized by a smoother shape. In the turbulent case, the decrease in the average settling time (at growing $u_{r m s}$ ) is associated with a progressive broadening of $P\left(t_{s}\right)$, in analogy to the behaviour of the cellular flow simulations.

To verify that peaked PDFs are associated to preferential concentration, we repeated the simulations with a cellular flow composed of 10 spatially dephased components. This modification has been sufficient to destroy the peaks and to smoothen the exit time distributions.

Conclusions. - We studied the settling of a diluted suspension of inertial particles. In particular we addressed the problem of the influence of flow structures on the settling speed. We focused on the case of very heavy particles, where the relevant parameters are the Stokes and Froude numbers St and Fr. We presented an heuristic argument predicting a quadratic decrease of the sedimentation speed $V_{s}$ with the typical velocity of the flow, in the regime of strong inertia $\left(\mathrm{Fr}^{2} / \mathrm{St} \gg 1\right)$. Such argument requires the assumption of almost vertical trajectories, which is valid in the case of weak, steady cellular flow. At larger flow velocities, different paths are favoured, which select mainly down-flow regions, thus increasing the settling velocity. This mechanism can be made less effective or even destroyed by a random dephasing or periodic perturbations of the cellular field on time scales smaller than the Stokes time of the particles. To further test the robustness of this scenario we repeated the simulations in a 2D turbulent flow. Even if the velocity field was smooth and dominated by the large scales, no systematic decrease in $V_{s}$ could be seen, while we still measured an increase with $U_{0}$. We can therefore argue that, in this case, while the spatial and temporal scales of the flow were large enough to allow the particles to find a favourable settling path that increases their speed, the slowing-down mechanism was suppressed because of the spatio-temporal chaoticity typical of turbulence. One can see a qualitative analogy between the suppression of the relenting regime in turbulence and in cellular flows which evolve rapidly enough in time.

We thank M. CEncini for a critical reading of the manuscript and useful suggestions.

\section{REFERENCES}

[1] Dibble R. W., Warnatz J. and Maas U., Combustion: Physical and Chemical Fundamentals, Modelling and Simulations, Experiments, Pollutant Formation (Springer, New York) 1996.

[2] Pruppacher H. R. and Klett J. D., Microphysics of Clouds and Precipitation (Kluwer Academic Publisher, The Netherlands) 2003.

[3] Shaw R. A., Annu. Rev. Fluid Mech., 35 (2003) 183.

[4] Squires K. D. and Eaton J. K., Phys. Fluids A, 3 (1991) 1169.

[5] Balkovsky E., Falkovich G. and Fouxon A., Phys. Rev. Lett., 86 (2001) 2790.

[6] BeC J., J. Fluid Mech., 528 (2005) 255.

[7] Duncan K., Mehlig B., Östlund S. and Wilkinson M., Phys. Rev. Lett., 95 (2005) 240602.

[8] Fessler J. R., Kulick J. D. and Eaton J. K., Phys. Fluids, 6 (1994) 3742.

[9] Wood A. M., Hwang W. and Eaton J. K., Int. J. Multiphase Flow, 31 (2005) 1220.

[10] Nishikawa T., Toroczkai Z., Grebogi C. and Tél T., Phys. Rev. E, 65 (2002) 026212.

[11] Falkovich G. and Pumir A., Phys. Fluids, 16 (2004) L47.

[12] Boffetta G., De Lillo F. and Gamba A., Phys. Fluids, 16 (2004) L20.

[13] Bec J., Biferale L., Cencini M., Lanotte A., Musacchio S. and Toschi F., Phys. Rev. Lett., 98 (2007) 084502.

[14] Bec J., Biferale L., Boffetta G., Celani A., Cencini M., Lanotte A., Musacchio S. and Toschi F., J. Fluid Mech., 550 (2006) 349.

[15] Vilela R. D., TÉl T., De Moure A. P. S. and Grebogi C., Phys. Rev. E, 75 (2007) 065203.

[16] Ruiz J., Macias D. and Peters F., Proc. Natl. Acad. Sci. U.S.A., 101 (2004) 17720.

[17] Stommel H., J. Mar. Res., 8 (1949) 2429.

[18] Maxey M. R. and Corrsin S., J. Atmos. Sci., 43 (1986) 1112.

[19] Solomon T. H. and Gollub J. P., Phys. Rev. A, 38 (1988) 6280.

[20] Maxey M. R., Phys. Fluids, 30 (1987) 1915.

[21] Marchioli C., Fantoni M. and Soldati A., Phys. Fluids, 19 (2007) 098101.

[22] Wang L. P. and Maxey M. R., J. Fluid Mech., 256 (1993) 27.

[23] YANG C. Y. and Lei U., J. Fluid Mech., 371 (1998) 179.

[24] Aliseda A., Cartellier A., Hainaux F. and Lasheras J. C., J. Fluid Mech., 468 (2002) 77.

[25] Yang T. S. and Shy S. S., Phys. Fluids, 15 (2003) 868.

[26] Pasquero C., Provenzale A. and Spiegel E. A., Phys. Rev. Lett., 91 (2003) 054502.

[27] Maxey M. R. and Riley J. J., Phys. Fluids, 26 (1983) 883.

[28] Castiglione P., Mazzino A., Muratore-GinanneSCHi P. and Vulpiani A., Physica D, 134 (1999) 75.

[29] Kraichnan R., Phys. Fluids, 10 (1967) 1417.

[30] Boffetta G., Celani A. and Vergassola M., Phys. Rev. E, 61 (2000) R29.

[31] Martins Afonso M., J. Phys. A: Math. Theor., 41 (2008) 385501. 